\title{
The Stochastic Solute Transport Model in 2-Dimensions
}

\subsection{Introduction}

In Chapter 6, we developed the generalised Stochastic Solute Transport Model (SSTM) in 1dimension and showed that it can model the hydrodynamic dispersion in porous media for the flow lengths ranging from 1 to $10000 \mathrm{~m}$. For computational efficiency, we have employed one of the fastest converging kernels tested in Chapter 6 for illustrative purposes, but, in principle, the SSTM should provide scale independent behaviour for any other velocity covariance kernel. If the kernel is developed based on the field data, then the SSTM based on that particular kernel should give realistic outputs from the model for that particular porous medium. In the development of the SSTM, we assumed that the hydrodynamic dispersion is one dimensional but by its very nature, the dispersion lateral to the flow direction occurs. We intend to explore this aspect in this chapter.

First, we solve the integral equation with the covariance kernel in two dimensions, and use the eigen values and functions thus obtained in developing the two dimensional stochastic solute transport model (SSTM2d). Then we solve the SSTM2d numerically using a finite difference scheme. In the last section of the chapter, we illustrate the behaviours of the SSTM2d graphically to show the robustness of the solution.

\subsection{Solving the Integral Equation}

We consider the flow direction to be $x$ and the coordinate perpendicular to $x$ to be $y$ in the 2 dimensional flow with in the porous matrix saturated with water. Then the distance between the points $\left(x_{1}, y_{1}\right)$ and $\left(x_{2}, y_{2}\right), r$, is given by $\left[\left(x_{1}-x_{2}\right)^{2}+\left(y_{1}-y_{2}\right)^{2}\right]^{1 / 2}$. We can then define a velocity covariance kernel as follows:

$$
q\left(x_{1}, y_{1}, x_{2}, y_{2}\right)=\sigma^{2} \exp \left[-\frac{r^{2}}{b}\right],
$$

where $\sigma^{2}$ is a constant. $\sigma^{2}$ is the variance at a given point, i.e., when $x_{1}=x_{2}$ and $y_{1}=y_{2}$. The covariance can be written as,

$$
\begin{aligned}
q\left(x_{1}, y_{1}, x_{2}, y_{2}\right) & =\sigma^{2} \exp \left[-\frac{\left[\left(x_{1}-x_{2}\right)^{2}+\left(y_{1}-y_{2}\right)^{2}\right]}{b}\right] \\
& =\sigma^{2} \exp \left[-\frac{\left(x_{1}-x_{2}\right)^{2}}{b}\right] \exp \left[-\frac{\left(y_{1}-y_{2}\right)^{2}}{b}\right]
\end{aligned}
$$


Then the integral equation can be written for 2 dimensions,

$$
\sigma^{2} \iint_{0}^{1} \exp \left[-\frac{\left(x_{1}-x_{2}\right)^{2}}{b}\right] \exp \left[-\frac{\left(y_{1}-y_{2}\right)^{2}}{b}\right] f\left(x_{2}, y_{2}\right) d x_{2} d y_{2}=\lambda f\left(x_{1}, y_{1}\right)
$$

where $f(x, y)$ and $\lambda$ are eigen functions and corresponding eigen values, respectively.

The covariance kernel is the multiplication of a function of $x$ and a function of $y$, and from the symmetry of equation (7.2.3), we can assume that the eigen function is the multiplication of a function of $x$ and a function of $y$ :

$$
f(x, y)=f_{x}(x) f_{y}(y)
$$

Then the integral equation can be written as,

$$
\begin{gathered}
\sigma^{2} \int_{0}^{1} \int_{0}^{1}\left(f_{x} e^{-\frac{\left(x_{1}-x_{2}\right)^{2}}{b}} d x_{2}\right)\left(f_{y} e^{-\frac{\left(y_{1}-y_{2}\right)^{2}}{b}} d y_{2}\right)=\lambda f_{x} f_{y}, \text { and } \\
\left\{\int_{0}^{1}\left(f_{x} e^{-\frac{\left(x_{1}-x_{2}\right)^{2}}{b}} d x_{2}\right)\right\}\left\{\int_{0}^{1}\left(f_{y} e^{-\frac{\left(y_{1}-y_{2}\right)^{2}}{b}} d y_{2}\right)\right\}=\frac{\lambda}{\sigma^{2}} f_{x} f_{y} .
\end{gathered}
$$

Therefore, if

$$
\begin{gathered}
\int_{0}^{1} f_{x} e^{-\frac{\left(x_{1}-x_{2}\right)^{2}}{b}} d x_{2}=\lambda_{x} f_{x}\left(x_{1}\right), \text { and } \\
\int_{0}^{1} f_{y} e^{-\frac{\left(y_{1}-y_{2}\right)^{2}}{b}} d y_{2}=\lambda_{y} f_{y}\left(y_{1}\right)
\end{gathered}
$$

Then we can see, $f(x, y)=f_{x} f_{y}$, and $\lambda=\sigma^{2} \lambda_{x} \lambda_{y}$.

This shows that we can use the eigen functions and eigen values obtained for 1-dimensional covariance kernels in Chapter 4 can be used in constructing the eigen functions and eigen values for two dimensional covariance kernel given in equation (7.2.2). Once we have obtained eigen functions and eigen values as solutions of the integral equation, we can derive the two dimensional mass conservation equation for solutes.

\subsection{Derivation of Mass Conservation Equation}

Consider the two dimensional infinitesimal volume element depicted in Figure 7.1. We can write the mass balance for solutes with in the element as,

$$
\begin{aligned}
\Delta C(x, y, t) n_{e} l \Delta x \Delta y= & \left\{J_{x}(x, y, t)-J_{x}(x+\Delta x, y, t)\right\} l \Delta y n_{e} \Delta t \\
& +\left\{J_{y}(x, y, t)-J_{y}(x, y+\Delta y, t)\right\} l \Delta x n_{e} \Delta t
\end{aligned}
$$




$$
\text { and } \frac{\Delta C(x, y, t)}{\Delta t}=\frac{\left(J_{x}-J_{x+\Delta x}\right)}{\Delta x}+\frac{\left(J_{y}-J_{y+\Delta y}\right)}{\Delta y},
$$

where $C(x, y, t)$ is the solute concentration and $J$ represents the solute flux at the location indicated by a subscript. We can expand $J$ using Taylor expansions as follows:

$J_{x+\Delta x}-J_{x}=\frac{1}{1 !} \frac{\partial J_{x}}{\partial x} \Delta x+\frac{1}{2 !} \frac{\partial^{2} J_{x}}{\partial x^{2}}(\Delta x)^{2}+\frac{1}{3 !} \frac{\partial^{3} J_{x}}{\partial x^{3}}(\Delta x)^{3}+$ higher order terms, and $J_{y+\Delta y}-J_{y}=\frac{1}{1 !} \frac{\partial J_{y}}{\partial y} \Delta y+\frac{1}{2 !} \frac{\partial^{2} J_{y}}{\partial y^{2}}(\Delta y)^{2}+\frac{1}{3 !} \frac{\partial^{3} J_{y}}{\partial y^{3}}(\Delta y)^{3}+$ higher order terms.

Lumping the higher order terms greater than 2, and denoting $R_{x}$ and $R_{y}$ as the remainders of the series,

$$
\begin{aligned}
& J_{x+\Delta x}-J_{x}=\frac{\partial J_{x}}{\partial x} \Delta x+\frac{1}{2 !} \frac{\partial^{2} J_{x}}{\partial x^{2}}(\Delta x)^{2}+R_{x}(\varepsilon), \text { and } \\
& J_{y+\Delta y}-J_{y}=\frac{\partial J_{y}}{\partial y} \Delta y+\frac{1}{2 !} \frac{\partial^{2} J_{y}}{\partial y^{2}}(\Delta y)^{2}+R_{y}(\varepsilon) .
\end{aligned}
$$

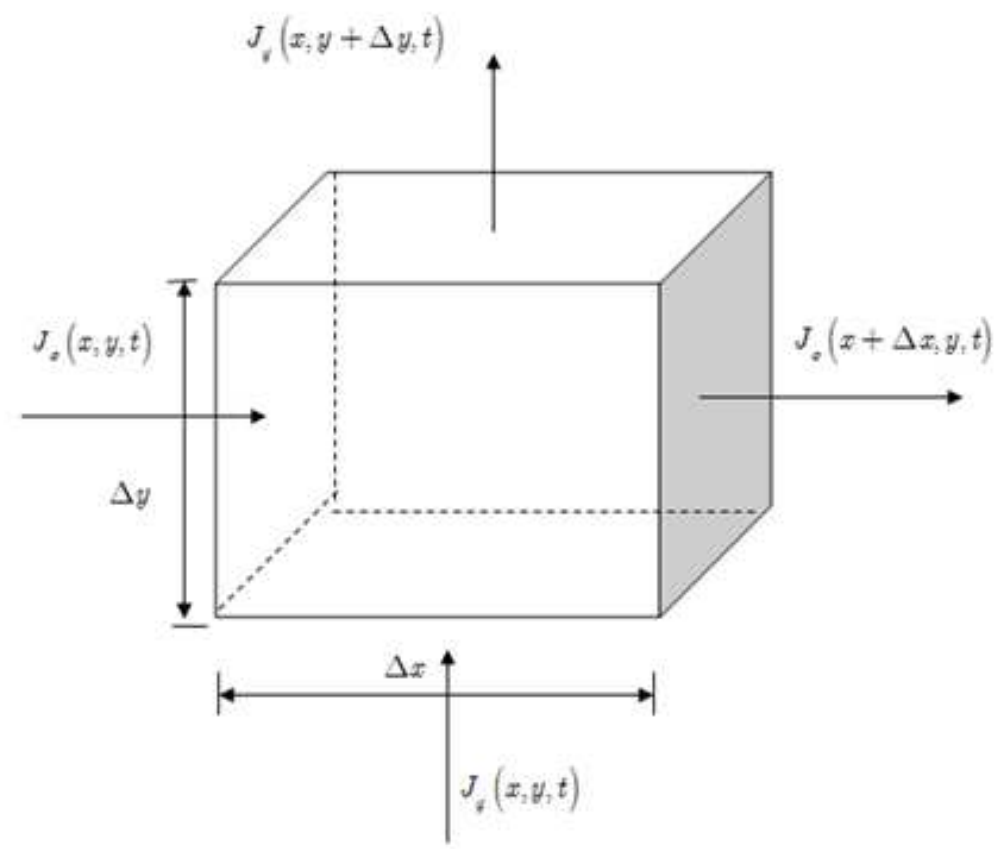

Figure 7.1. Two dimensional infinitesimal volume element with a depth $l$ and porosity $n_{e} . \Delta x$ and $\Delta y$ are side lengths in $x$ and $y$ directions, respectively. 
Substituting equations (7.3.2a) and (7.3.2b) back to equation (7.3.1) and taking the limit $\Delta t \rightarrow 0$,

$$
\begin{aligned}
\frac{\partial C(x, y, t)}{\partial t} & =-\frac{\partial J_{x}}{\partial x}-\frac{1}{2} \frac{\partial^{2} J_{x}}{\partial x^{2}} \Delta x-\frac{\partial J_{y}}{\partial y}-\frac{1}{2} \frac{\partial^{2} J_{y}}{\partial y^{2}} \Delta y+R_{x}^{\prime}(\varepsilon) \\
& =-\left(\frac{\partial J_{x}}{\partial x}+\frac{\partial J_{y}}{\partial y}\right)-\frac{h_{x}}{2}\left(\frac{\partial^{2} J_{x}}{\partial x^{2}}\right)-\frac{h_{y}}{2}\left(\frac{\partial^{2} J_{y}}{\partial y^{2}}\right)+R_{x}^{\prime}(\varepsilon)+R_{y}^{\prime}(\varepsilon)^{\prime}
\end{aligned}
$$

where $h_{x}=\Delta x$ and $h_{y}=\Delta y$.

$$
d C=-\left[\frac{\partial J_{x}}{\partial x}+\frac{h_{x}}{2} \frac{\partial^{2} J_{x}}{\partial x^{2}}\right] d t-\left[\frac{\partial J_{y}}{\partial y}+\frac{h_{y}}{2} \frac{\partial^{2} J_{y}}{\partial y^{2}}\right] d t+\left(R_{x}^{\prime}(\varepsilon)+R_{y}^{\prime}(\varepsilon)\right) d t
$$

$\operatorname{Assuming}\left(R_{x}^{\prime}(\varepsilon)+R_{y}^{\prime}(\varepsilon)\right) d t \approx 0$

$$
d C(x, y, t)=-\left[\frac{\partial J_{x}}{\partial x}+\frac{h_{x}}{2} \frac{\partial^{2} J_{x}}{\partial x^{2}}\right] d t-\left[\frac{\partial J_{y}}{\partial y}+\frac{h_{y}}{2} \frac{\partial^{2} J_{y}}{\partial y^{2}}\right] d t
$$

Now we can express the solute flux in terms of solute concentration and velocity,

$$
\begin{aligned}
& J_{x}(x, y, t)=V_{x}(x, y, t) C(x, y, t), \text { and } \\
& J_{y}(x, y, t)=V_{y}(x, y, t) C(x, y, t) .
\end{aligned}
$$

We can express the velocity in terms of the mean velocity vector and a noise vector,

$$
\underline{V}(x, y, t)=\underline{\bar{V}}(x, y, t)+\underline{\xi}(x, y, t),
$$

where $\underline{V}(x, y, t), \underline{\bar{V}}(x, y, t)$ and $\underline{\xi}(x, y, t)$ are velocity, mean velocity and noise vectors respectively. Instantaneous velocity vector can now be expressed as,

$$
\underline{V}(x, y, t)=V_{x}(x, y, t) \underline{i}+V_{y}(x, y, t) \underline{j},
$$

where $\underline{i}$ and $\underline{j}$ are unit vectors in $x$ and $y$ directions, respectively; and, $V_{x}(x, y, t)$ and $V_{y}(x, y, t)$ are the magnitudes of the velocities in $x$ and $y$ directions. By substituting the vector components in equation (7.3.6) in to equation (7.3.7), we obtain,

$$
\begin{aligned}
\underline{V}(x, y, t) & =\left(\bar{V}_{x}(x, y, t)+\xi_{x}(x, y, t)\right) \underline{i}+\left(\bar{V}_{y}(x, y, t)+\xi_{y}(x, y, t)\right) \underline{j} \\
& =\left(\bar{V}_{x} \underline{i}+\bar{V}_{y} \underline{j}\right)+\left(\xi_{x}(x, y, t) \underline{i}+\xi_{y}(x, y, t) \underline{j}\right)
\end{aligned}
$$

where $\xi_{x}$ and $\xi_{y}$ are the noise components in $x$ and $y$ directions. We can see the noise term appearing as, $\left(\xi_{x}(x, y, t) \underline{i}+\xi_{y}(x, y, t) \underline{j}\right)=\underline{\xi}(x, y, t)$. 
To simplify the notation,

$$
\begin{gathered}
V_{x}=\bar{V}_{x}+\xi_{x}, \text { and } \\
V_{y}=\bar{V}_{y}+\xi_{y} .
\end{gathered}
$$

By substituting these equations in to equations (7.3.5a) and (7.3.5b), and then substituting the resulting equations in to equation (7.3.4), we obtain,

$$
d C=S_{x}\left(\bar{V}_{x} C\right) d t+S_{y}\left(\bar{V}_{y} C\right) d t+S_{x}\left(C_{x} \xi_{x}\right) d t+S_{y}\left(C_{y} \xi_{y}\right) d t
$$

where $S_{x}=-\left(\frac{\partial}{\partial x}+\frac{h_{x}}{2} \frac{\partial^{2}}{\partial x^{2}}\right)$, and

$$
S_{y}=-\left(\frac{\partial}{\partial y}+\frac{h_{y}}{2} \frac{\partial^{2}}{\partial y^{2}}\right) \text {. }
$$

We can now write,

$d C=\left(S_{x}\left(\bar{V}_{x} C\right)+S_{y}\left(\bar{V}_{y} C\right)\right) d t+S_{x}\left(C_{x} \xi_{x}\right) d t+S_{y}\left(C_{y} \xi_{y}\right) d t$, and bringing $d t$ in to the parenthesis in the third and fourth terms of the right hand side,

$$
d C=\left(S_{x}\left(\bar{V}_{x} C\right)+S_{y}\left(\bar{V}_{y} C\right)\right) d t+S_{x}\left(C \xi_{x} d t\right)+S_{y}\left(C \xi_{y} d t\right) .
$$

As in the one dimensional case, we can define,

$\beta_{x}=\xi_{x} d t$ and $\beta_{y}=\xi_{y} d t$, and these are the components of a noise vector, $\beta$, which operates in a Hilbert space having eigen functions as co-ordinates. Equation (7.3.12) can now be expressed as,

$$
d C=\left(S_{x}\left(\bar{V}_{x} C\right)+S_{y}\left(\bar{V}_{y} C\right)\right) d t+S_{x}\left(C d \beta_{x}\right)+S_{y}\left(C d \beta_{y}\right)
$$

The resultant noise term is given by,

$$
d \beta=\sigma \sum_{j=1}^{m} \sqrt{\lambda_{x, j} \lambda_{y, j}} f_{x, j} f_{y, j} d b_{j}(t),
$$

where $f_{x, j}=$ eigen functions in $x$ direction, and

$$
f_{y, j}=\text { eigen functions in } y \text { direction. }
$$

Now we can express the components in $x$ and $y$ directions,

$$
\begin{gathered}
d \beta_{x}=d \beta \cos \theta, \text { and } \\
d \beta_{y}=d \beta \sin \theta .
\end{gathered}
$$


We make an assumption that $\theta$ is defined by $\cos \theta=\frac{x}{\sqrt{|x|^{2}+|y|^{2}}} ; \quad \sin \theta=\frac{y}{\sqrt{|x|^{2}+|y|^{2}}}$. This is a simplifying approximation which makes the modelling more tractable; as the noise term is quite random, this approximation does not make significant difference to final results.

Then

$$
d C=\left(S_{x}\left(\bar{V}_{x} C\right)+S_{y}\left(\bar{V}_{y} C\right)\right) d t+S_{x}(C(x, y, t) d \beta \cos \theta)+S_{y}(C(x, y, t) d \beta \sin \theta) .
$$

Analogous to equation (4.2.4),

$$
\begin{aligned}
S_{x}(C(x, y, t) d \beta \cos \theta) & =S_{x}\left(C(x, y, t)\left\{\sigma \sum_{j=1}^{m} \sqrt{\lambda_{x, j} \lambda_{y, j}} f_{x, j} f_{y, j} d b_{j}(t)\right\} \cos \theta\right) . \\
-S_{x}(C d \beta \cos \theta) & =-\sigma \sum_{j=1}^{m} \sqrt{\lambda_{x, j} \lambda_{y, j}} f_{y, j} S_{x}\left(C f_{x, j} \cos \theta\right) d b_{j}(t) \\
= & \sigma \sum_{j=1}^{m} \sqrt{\lambda_{x, j} \lambda_{y, j}} f_{y, j}\left\{-S_{x}\left(C f_{x, j} \cos \theta\right)\right\} d b_{j}(t) .
\end{aligned}
$$

Now we can expand the terms in the brackets in equation (7.3.18),

$$
-S_{x}\left(C f_{x, j} \cos \theta\right)=\left(\frac{\partial}{\partial x}+\frac{h_{x}}{2} \frac{\partial^{2}}{\partial x^{2}}\right)\left(C f_{x, j} \cos \theta\right) .
$$

We see that,

$$
\begin{aligned}
\frac{\partial}{\partial x}\left(C f_{x, j} \cos \theta\right) & =C f_{x, j} \frac{\partial \cos \theta}{\partial x}+C \cos \theta \frac{\partial f_{x, j}}{\partial x}+f_{x, j} \cos \theta \frac{\partial C}{\partial x}, \text { and } \\
\frac{\partial^{2}}{\partial x^{2}}\left(C f_{x, j} \cos \theta\right) & =\left(C f_{x, j} \frac{\partial^{2} \cos \theta}{\partial x^{2}}+C \frac{\partial \cos \theta}{\partial x} \frac{\partial f_{x, j}}{\partial x}+f_{x, j} \frac{\partial \cos \theta}{\partial x} \frac{\partial C}{\partial x}\right) \\
& +\left(C \cos \theta \frac{\partial^{2} f_{x, j}}{\partial x^{2}}+C \frac{\partial \cos \theta}{\partial x} \frac{\partial f_{x, j}}{\partial x}+\frac{\partial f_{x, j}}{\partial x} \cos \theta \frac{\partial C}{\partial x}\right) \\
& +\left(f_{x, j} \cos \theta \frac{\partial^{2} C}{\partial x^{2}}+f_{x, j} \frac{\partial \cos \theta}{\partial x} \frac{\partial C}{\partial x}+\cos \theta \frac{\partial f_{x, j}}{\partial x} \frac{\partial C}{\partial x}\right)
\end{aligned}
$$


Now,

$$
\begin{aligned}
& -S_{x}\left(C f_{x, j} \cos \theta\right) \\
& =C(x, y, t)\left[f_{x, j} \frac{\partial \cos \theta}{\partial x}+\cos \theta \frac{\partial f_{x, j}}{\partial x}+\frac{h_{x}}{2}\left(f_{x, j} \frac{\partial^{2} \cos \theta}{\partial x^{2}}+\frac{\partial \cos \theta}{\partial x} \frac{\partial f_{x, j}}{\partial x}+\cos \theta \frac{\partial^{2} f_{x, j}}{\partial x^{2}}+\frac{\partial \cos \theta}{\partial x} \frac{\partial f_{x, j}}{\partial x}\right)\right] \\
& +\frac{\partial C(x, y, t)}{\partial x}\left[f_{x, j} \cos \theta \frac{\partial f_{x, j}}{\partial x}+\frac{h_{x}}{2}\left(f_{x, j} \frac{\partial \cos \theta}{\partial x}+\frac{\partial f_{x, j}}{\partial x} \cos \theta+f_{x, j} \frac{\partial \cos \theta}{\partial x}+\cos \theta \frac{\partial f_{x, j}}{\partial x}\right)\right] \\
& +\frac{\partial^{2} C(x, y, t)}{\partial x^{2}}\left[\frac{h_{x}}{2}\left(f_{x, j} \cos \theta\right)\right]
\end{aligned}
$$

Then,

$$
\begin{aligned}
& -S_{x}\left(C f_{x, j} \cos \theta\right) \\
& =C(x, y, t)\left\{\left(f_{x, j} \frac{\partial \cos \theta}{\partial x}+\cos \theta \frac{\partial f_{x, j}}{\partial x}\right)+\frac{h_{x}}{2}\left(f_{x, j} \frac{\partial^{2} \cos \theta}{\partial x^{2}}+2 \frac{\partial \cos \theta}{\partial x} \frac{\partial f_{x, j}}{\partial x}+\cos \theta \frac{\partial^{2} f_{x, j}}{\partial x^{2}}\right)\right\} \\
& +\frac{\partial C(x, y, t)}{\partial x}\left\{f_{x, j} \cos \theta+\frac{h_{x}}{2}\left(2 f_{x, j} \frac{\partial \cos \theta}{\partial x}+2 \cos \theta \frac{\partial f_{x, j}}{\partial x}\right)\right\} \\
& +\frac{\partial^{2} C(x, y, t)}{\partial x^{2}}\left\{\frac{h_{x}}{2} f_{x, j} \cos \theta\right\} .
\end{aligned}
$$

Simplifying, we obtain,

$$
\begin{aligned}
-S_{x}\left(C f_{x, j} \cos \theta\right) & =C(x, y, t)\left\{\frac{\partial\left(f_{x, j} \cos \theta\right)}{\partial x}+\frac{h_{x}}{2} \frac{\partial^{2}\left(f_{x, j} \cos \theta\right)}{\partial x^{2}}\right\} \\
& +\frac{\partial C(x, y, t)}{\partial x}\left\{f_{x, j} \cos \theta+h_{x} \frac{\partial\left(f_{x, j} \cos \theta\right)}{\partial x}\right\} \\
& +\frac{\partial^{2} C(x, y, t)}{\partial x^{2}}\left\{\frac{h_{x}}{2}\left(f_{x, j} \cos \theta\right)\right\} .
\end{aligned}
$$

Similarly,

$$
\begin{aligned}
& S_{y}\left(C f_{y, j} \cos \theta\right) \\
& =C(x, y, t)\left\{\frac{\partial\left(f_{y, j} \sin \theta\right)}{\partial y}+\frac{h_{x}}{2} \frac{\partial^{2}\left(f_{y, j} \sin \theta\right)}{\partial y^{2}}\right\}+\frac{\partial C(x, y, t)}{\partial y}\left\{f_{y, j} \sin \theta+h_{y} \frac{\partial\left(f_{y, j} \sin \theta\right)}{\partial y}\right\} \\
& +\frac{\partial^{2} C(x, y, t)}{\partial y^{2}}\left\{\frac{h_{y}}{2}\left(f_{y, j} \sin \theta\right)\right\} .
\end{aligned}
$$


$\therefore S_{x}(C d \beta \cos \theta)$

$$
=-\sigma \sum_{j=1}^{m} \sqrt{\lambda_{x, j} \lambda_{y, j}}\left\{\left(P_{0, y, j} C(x, y, t)+P_{1, y, j} \frac{\partial C(x, y, t)}{\partial x}+P_{2 x, y, j} \frac{\partial^{2} C(x, y, t)}{\partial x^{2}}\right)\right\},
$$

Where

$$
\begin{gathered}
P_{0, j}=P_{0, x, y, j}=f_{y, j}\left(\frac{\partial\left(f_{x, j} \cos \theta\right)}{\partial x}+\frac{h_{x}}{2} \frac{\partial^{2}\left(f_{x, j} \cos \theta\right)}{\partial x^{2}}\right) ; \\
P_{1, j}=P_{1, x, y, j}=f_{y, j}\left(f_{x, j} \cos \theta+h_{x} \frac{\partial\left(f_{x, j} \cos \theta\right)}{\partial x}\right) ; \text { and } \\
P_{2, j}=P_{2, x, y, j}=f_{y, j}\left(\frac{h_{x}}{2}\left(f_{x, j} \cos \theta\right)\right) .
\end{gathered}
$$

Similarly,

$$
\begin{gathered}
S_{y}(C d \beta \sin \theta)=-\sigma \sum_{j=1}^{m} \sqrt{\lambda_{x, j} \lambda_{y, j}}\left\{Q_{0, j} C(x, y, t)+Q_{1, j} \frac{\partial C(x, y, t)}{\partial y}+Q_{2, j} \frac{\partial^{2} C(x, y, t)}{\partial y^{2}}\right\} \\
Q_{0, j}=f_{x, j}\left(\frac{\partial\left(f_{y, j} \sin \theta\right)}{\partial y}+\frac{h_{y}}{2} \frac{\partial^{2}\left(f_{y, j} \sin \theta\right)}{\partial y^{2}}\right) ; \\
Q_{1, j}=f_{x, j}\left(f_{y, j} \sin \theta+h_{y} \frac{\partial\left(f_{y, j} \sin \theta\right)}{\partial y}\right) ; \text { and } \\
\quad Q_{2, j}=f_{x, j}\left(\frac{h_{y}}{2}\left(f_{y, j} \sin \theta\right)\right) . \\
\therefore S_{x}\left(C d \beta_{x}\right)+S_{y}\left(C d \beta_{y}\right) \quad(7.3 \\
=-\sigma \sum_{j=1}^{m} \sqrt{\lambda_{x, j} \lambda_{y, j}}\left\{\left(P_{0 j}+Q_{0, j}\right) C(x, t)+\left(P_{1, j} \frac{\partial C}{\partial x}+Q_{1, j} \frac{\partial C}{\partial y}\right)+\left(P_{2, j} \frac{\partial^{2} C}{\partial x^{2}}+Q_{2, j} \frac{\partial^{2} C}{\partial y^{2}}\right)\right\} d b_{j}(t)
\end{gathered}
$$

Therefore,

$$
\begin{aligned}
d C= & -C(x, y, t) d I_{0 x}-\frac{\partial C}{\partial x} d I_{1 x}-\frac{\partial^{2} C}{\partial x^{2}} d I_{2 x}, \\
& -C(x, y, t) d I_{0 y}-\frac{\partial C}{\partial y} d I_{1 y}-\frac{\partial^{2} C}{\partial y^{2}} d I_{2 y} \\
\text { where } d I_{0 x}= & \left(\frac{\partial \bar{V}_{x}}{\partial x}+\frac{h_{x}}{2} \frac{\partial^{2} \bar{V}_{x}}{\partial x^{2}}\right) d t+\sigma \sum_{j=1}^{m} \sqrt{\lambda_{x, j} \lambda_{y, j}} P_{0 j} d b_{j}(t),
\end{aligned}
$$




$$
\begin{aligned}
& d I_{1 x}=\left(\bar{V}_{x}+h_{x} \frac{\partial \bar{V}_{x}}{\partial x}\right) d t+\sigma \sum_{j=1}^{m} \sqrt{\lambda_{x, j} \lambda_{y, j}} P_{1 j} d b_{j}(t), \\
& d I_{2 x}=\left(\frac{h_{x}}{2} \bar{V}_{x}\right) d t+\sigma \sum_{j=1}^{m} \sqrt{\lambda_{x, j} \lambda_{y, j}} P_{2 j} d b_{j}(t), \\
& d I_{0 y}=\left(\frac{\partial \bar{V}_{y}}{\partial y}+\frac{h_{y}}{2} \frac{\partial^{2} \bar{V}_{y}}{\partial y^{2}}\right) d t+\sigma \sum_{j=1}^{m} \sqrt{\lambda_{x, j} \lambda_{y, j}} Q_{0 j} d b_{j}(t), \\
& d I_{1 y}=\left(\bar{V}_{y}+h_{y} \frac{\partial \bar{V}_{y}}{\partial y}\right) d t+\sigma \sum_{j=1}^{m} \sqrt{\lambda_{x, j} \lambda_{y, j}} Q_{1 j} d b_{j}(t),
\end{aligned}
$$

and

$$
d I_{2 y}=\left(\frac{h_{y}}{2} \bar{V}_{y}\right) d t+\sigma \sum_{j=1}^{m} \sqrt{\lambda_{x, j} \lambda_{y, j}} Q_{2 j} d b_{j}(t)
$$

Equations (7.3.25) - (7.3.31) constitute the SSTM2d with the definitions for $P$ s and $Q$ s given by equations (7.3.19) to (7.3.24). The SSTM2d has similar Ito diffusions for velocities as in the one dimensional case. Equation (7.3.19) shows an elegant extension of SSTM into 2dimensions. It should be noted that the eigen values for both directions are the same for the $[0,1]$ domain, further simplifying the equations.

The development of the SSTM2d is based on the fact that any kernel can be expressed as a multiplication of two kernels, for example, as in equation (7.2.2); and we know the methodology of obtaining the eigen values and eigen functions for any kernel. Therefore, we can solve the SSTM2d for any kernel. However, for the illustrative purposes, we only focus on the kernel given in equation (7.2.2) in this chapter.

\subsubsection{A Summary of the Finite Difference Scheme}

To understand the behaviour of the SSTM2d, we need to solve the equations numerically by using a finite difference scheme developed for the purpose. We only highlight the pertinent equations in the algorithm.

Now let $x_{i}=i \Delta x, y_{j}=j \Delta y, t_{n}=n \Delta t$, and $C_{[i, j]}^{n}=C_{\left[x_{i}, y_{j}\right]}^{\left.t_{n}\right]}$, Equation (7.3.25) can be redisplayed as ,

$$
d c_{\left[x_{i}, y_{j}\right]}^{t_{n}}=d c_{[i, j]}^{n}=-c_{[i, j]}^{n} d I_{0, x_{i}}-\frac{d c_{[i, j]}^{n}}{d x_{i}} d I_{1, x_{i}}-\frac{d^{2} c_{[i, j]}^{n}}{d x_{i}^{2}} d I_{2, x_{i}}-c_{[i, j]}^{n} d I_{0, y_{j}}-\frac{d c_{[i, j]}^{n}}{d y_{j}} d I_{1, y_{j}}-\frac{d^{2} c_{[i, j]}^{n}}{d y_{j}{ }^{2}} d I_{2, y_{j}} .
$$

We use the forward difference to calculate the first first-order derivatives with respect to time $(t)$, the backward difference to calculate the first-order derivative in $x$ and $y$ directions and the central difference to calculate the second-order derivatives, i.e., 


$$
\begin{aligned}
& \frac{d c_{[i, j]}^{n}}{d t}=\frac{c_{[i, j]}^{n+1}-c_{[i, j]}^{n}}{\Delta t}, \frac{d c_{[i, j]}^{n}}{d x}=\frac{c_{[i, j]}^{n}-c_{[i-1, j]}^{n}}{\Delta x}, \frac{d c_{[i, j]}^{n}}{d y}=\frac{c_{[i, j]}^{n}-c_{[i, j-1]}^{n}}{\Delta y}, \\
& \frac{d^{2} c_{[i, j]}^{n}}{d x^{2}}=\frac{c_{[i+1, j]}^{n}-2 c_{[i, j]}^{n}+c_{[i-1, j]}^{n}}{\Delta x^{2}}, \frac{d^{2} c_{[i, j]}^{n}}{d x^{2}}=\frac{c_{[i, j+1]}^{n}-2 c_{[i, j]}^{n}+c_{[i, j-1]}^{n}}{\Delta y^{2}} .
\end{aligned}
$$

We can develop the finite difference scheme to solve the SSTM2d based on the following equation:

$$
\begin{aligned}
c_{[i, j]}^{n+1}-c_{[i, j]}^{n}= & -c_{[i, j]}^{n} d I_{0, x_{i}}-\left(\frac{c_{[i, j]}^{n}-c_{[i-1, j]}^{n}}{\Delta x}\right) d I_{1, x_{i}}-\left(\frac{c_{[i+1, j]}^{n}-2 c_{[i, j]}^{n}+c_{[i-1, j]}^{n}}{\Delta x^{2}}\right) d I_{2, x_{i}} \\
& -c_{[i, j]}^{n} d I_{0, y_{j}}-\left(\frac{c_{[i, j]}^{n}-c_{[i, j-1]}^{n}}{\Delta y}\right) d I_{1, y_{j}}-\left(\frac{c_{[i, j+1]}^{n}-2 c_{[i, j]}^{n}+c_{[i, j-1]}^{n}}{\Delta y^{2}}\right) d I_{2, y_{j}} .
\end{aligned}
$$

We illustrate some realisations of the solutions graphically in the next section.

\subsubsection{Graphical Depictions of Realisations}

In the following figures, we present a sample of solution realisations of the SSTM2d to illustrate the behaviours of the model under different parameter values for the boundary condition: $C(t, x, y)=1.0$ at $(x=0.0$ and $y=0.0)$ for any given $t$. The value of $b$ is kept at 0.1 for all computations.

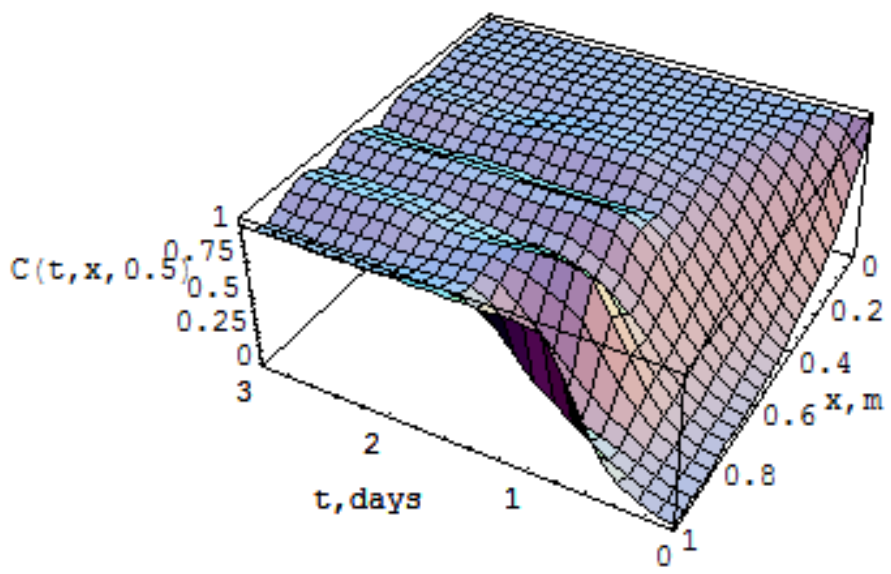

Figure 7.2. A realisation of concentration at $\mathrm{y}=0.5 \mathrm{~m}$ when $\sigma^{2}=0.0001$. 


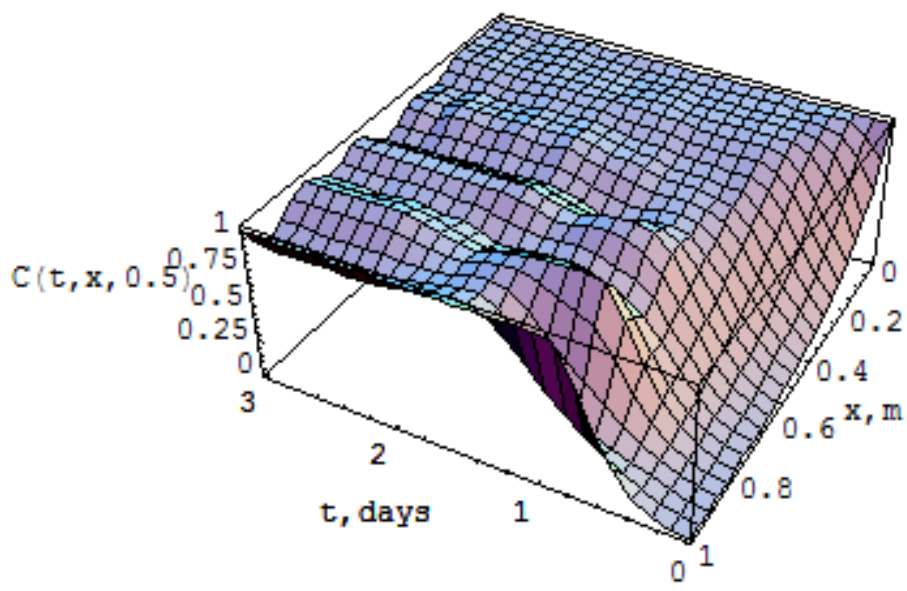

Figure 7.3. A realisation of concentration at $y=0.5 \mathrm{~m}$ when $\sigma^{2}=0.001$.

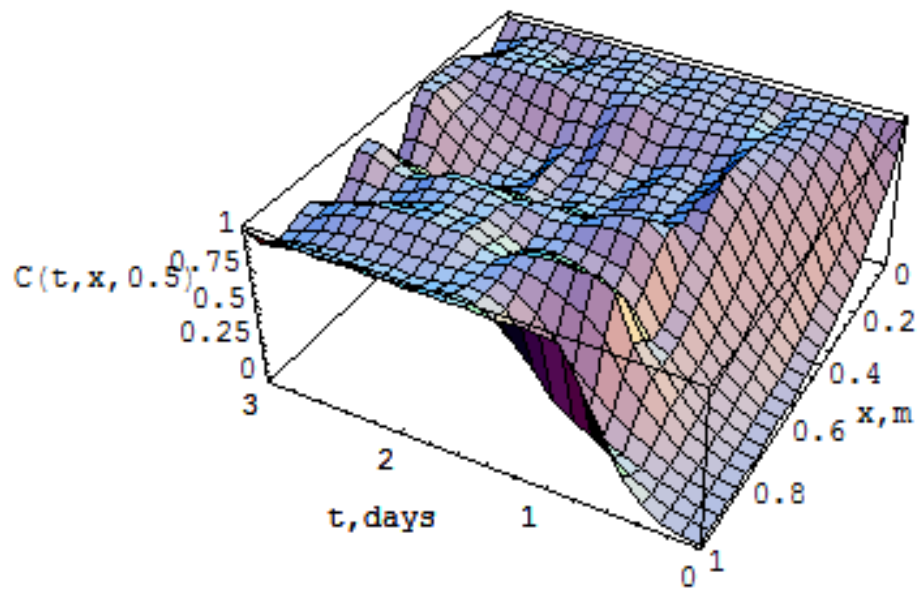

Figure 7.4. A realisation of concentration at $y=0.5 \mathrm{~m}$ when $\sigma^{2}=0.01$. 


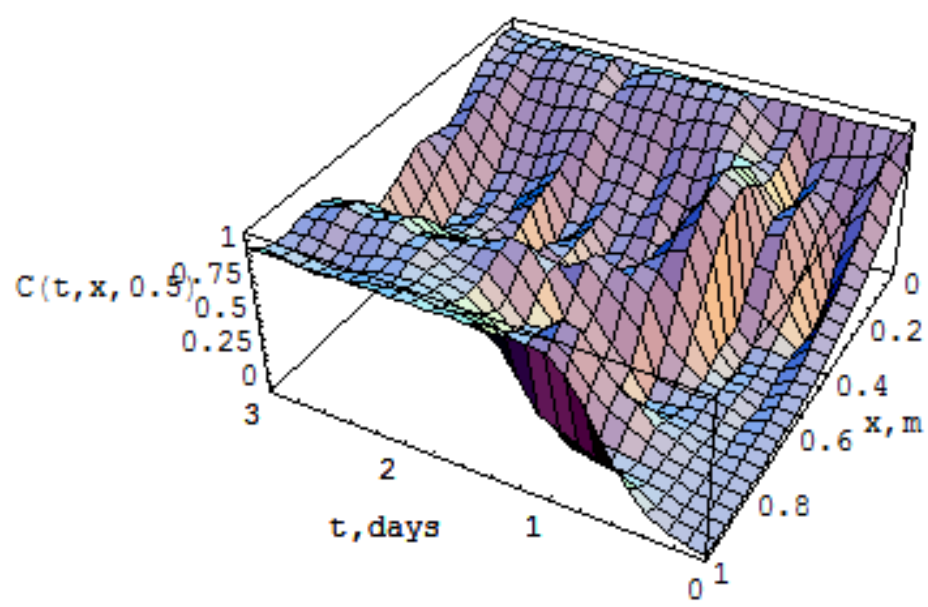

Figure 7.5. A realisation of concentration at $y=0.5 \mathrm{~m}$ when $\sigma^{2}=0.1$

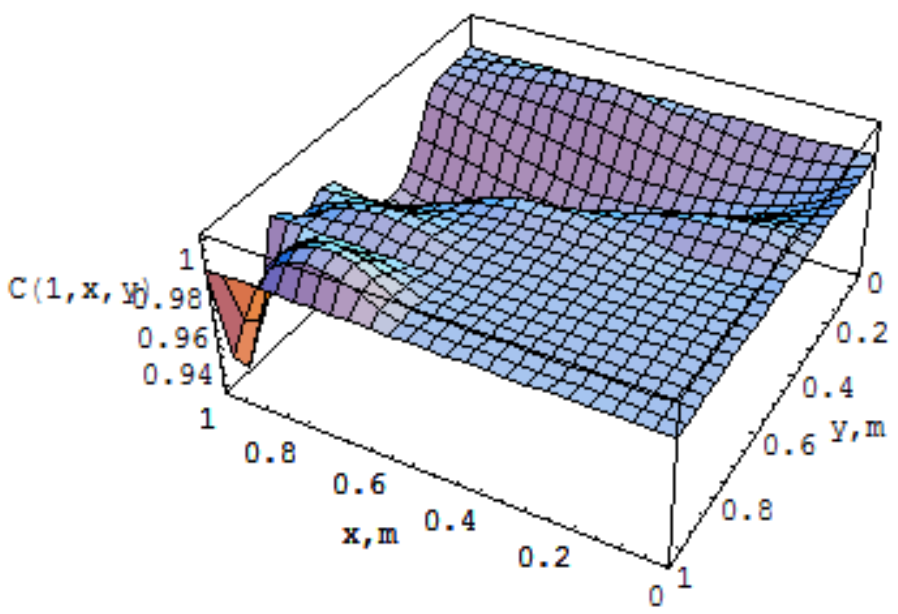

Figure 7.6. A realisation of concentration at $t=1$ day when $\sigma^{2}=0.0001$. 


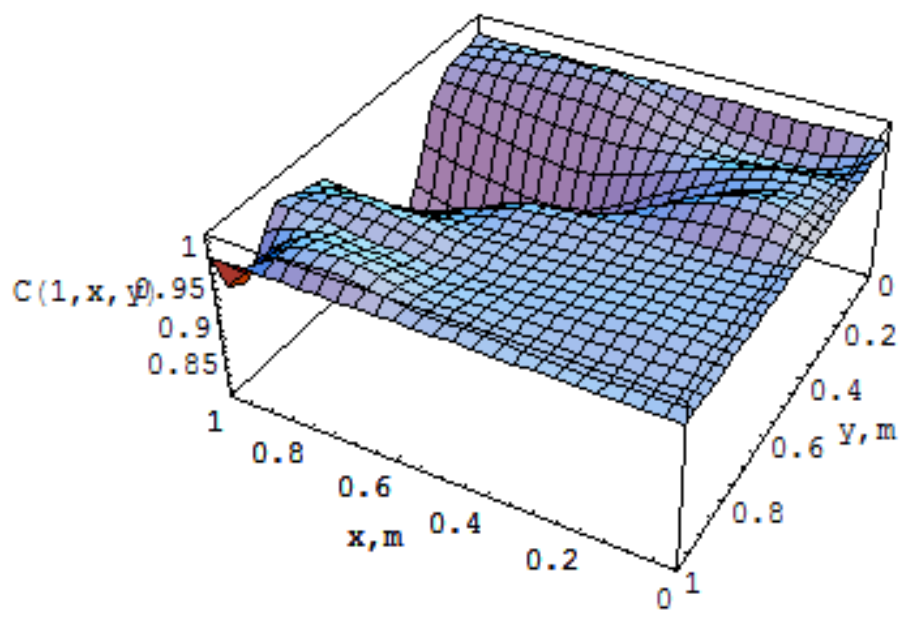

Figure 7.7. A realisation of concentration at $t=1$ day when $\sigma^{2}=0.001$.

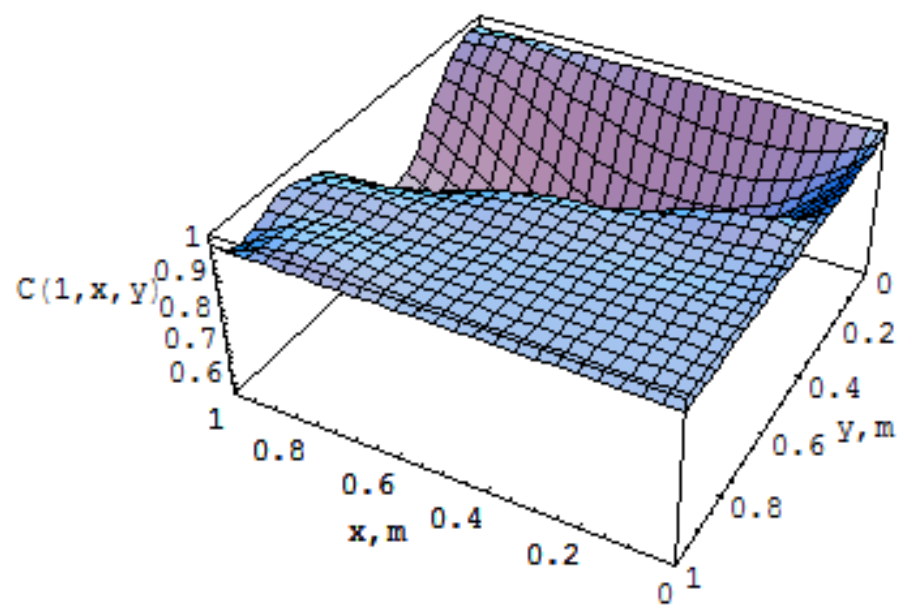

Figure 7.8. A realisation of concentration at $t=1$ day when $\sigma^{2}=0.01$. 


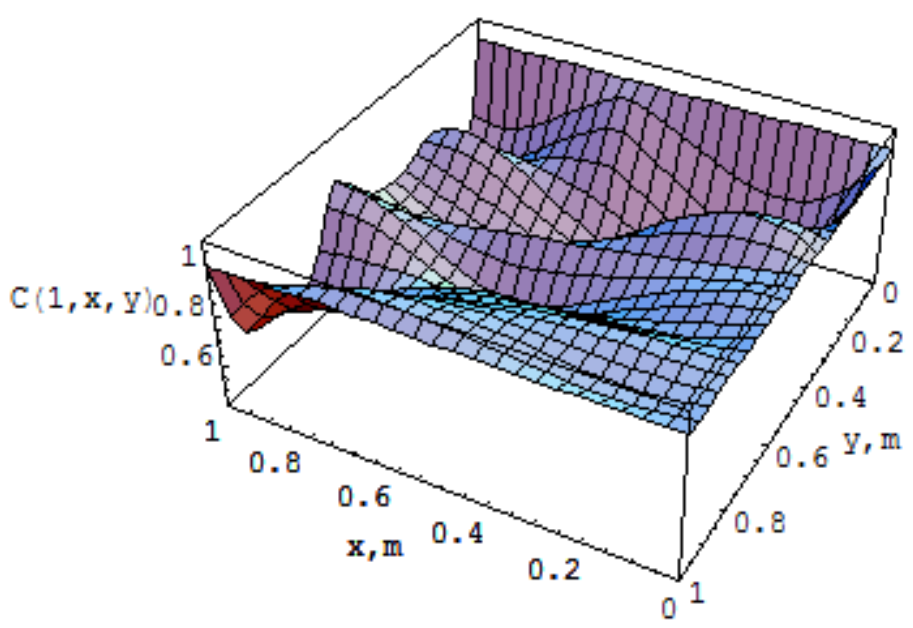

Figure 7.9. A realisation of concentration at $t=1$ day when $\sigma^{2}=0.1$.

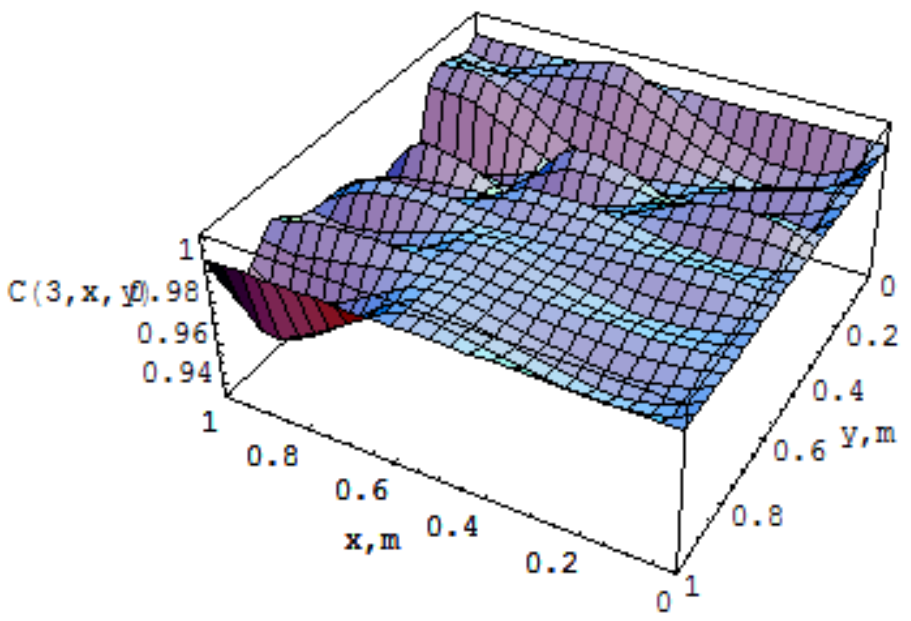

Figure 7.10. A realisation of concentration at $t=3$ days when $\sigma^{2}=0.0001$. 


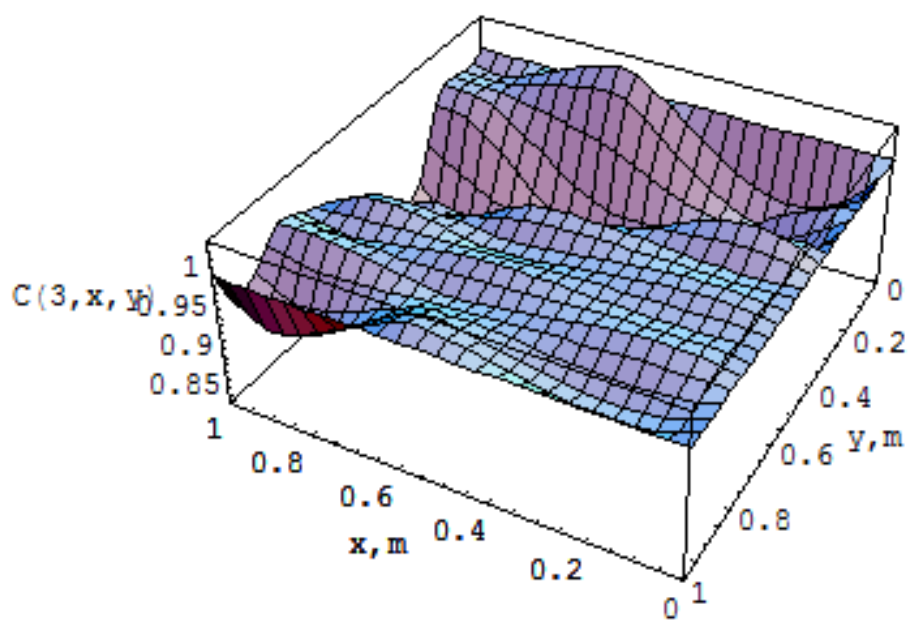

Figure 7.11. A realisation of concentration at $t=3$ days when $\sigma^{2}=0.001$.

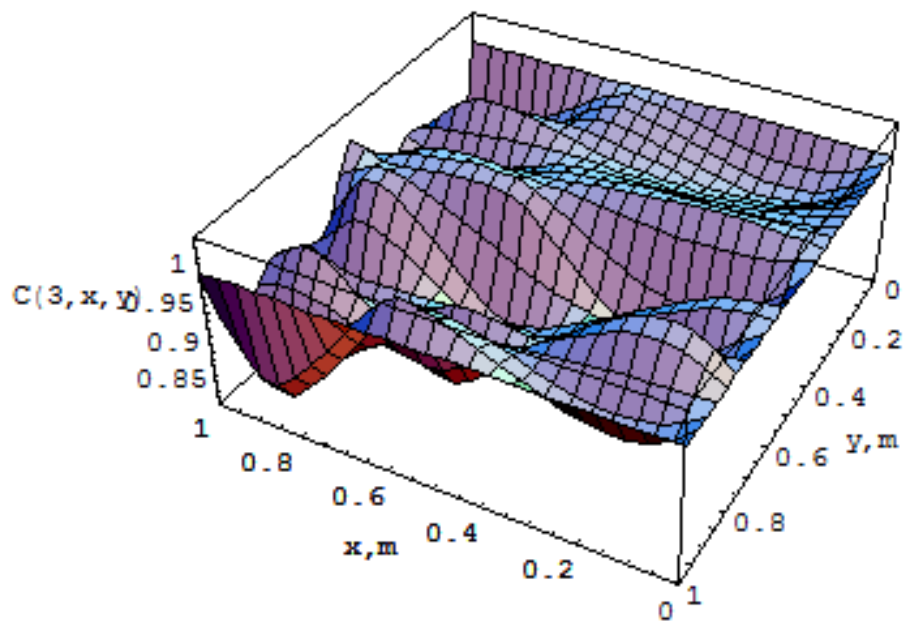

Figure 7.12. A realisation of concentration at $t=3$ days when $\sigma^{2}=0.01$. 


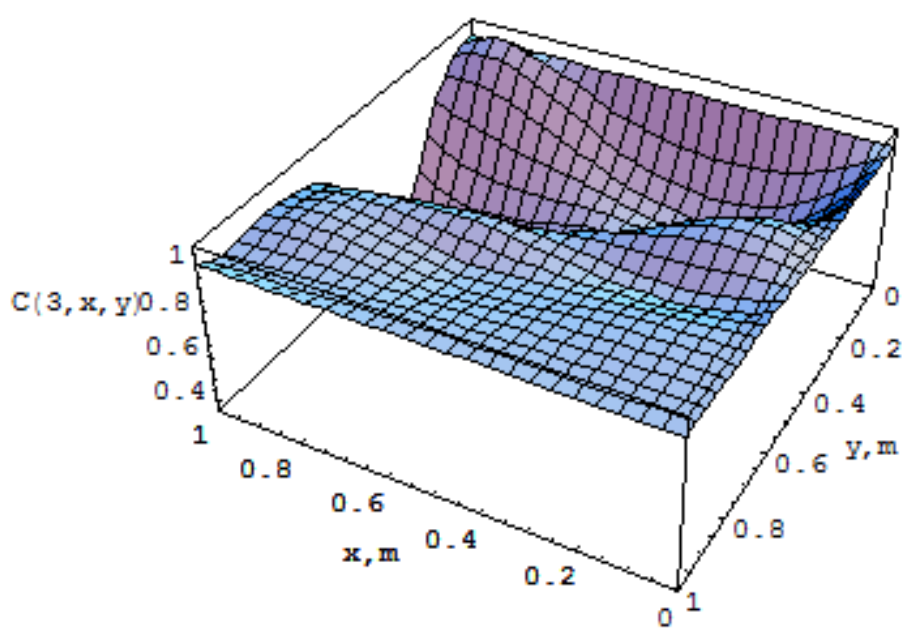

Figure 7.13. A realisation of concentration at $t=3$ days under $\sigma^{2}=0.1$.

The figures above shows that the numerical scheme is robust to obtain the concentration realisations for a range of values of $\sigma^{2}$. As $\sigma^{2}$ increases the stochasticity of the realisations increases.

\subsection{Longitudinal and Transverse Dispersivity according to SSTM2D}

To estimate the longitudinal and transverse dispersivities, we start with the partial differential equation for advection and dispersion, taking $x$ axis to be the direction of the flow.

The two-dimensional advection-dispersion equation can be written as,

$$
\frac{\partial C}{\partial t}=\left\{D_{L}\left(\frac{\partial^{2} C}{\partial x^{2}}\right)+D_{T}\left(\frac{\partial^{2} C}{\partial y^{2}}\right)\right\}-v_{x}\left(\frac{\partial C}{\partial x}\right)
$$

where $C=$ solution concentration $(\mathrm{mg} / \mathrm{l})$,

$t=$ time (day),

$D_{L}=$ hydrodynamic dispersion coefficient parallel to the principal direction of flow (longitudinal) (m²/day),

$D_{T}=$ hydrodynamic dispersion coefficient perpendicular to the principal direction of flow (transverse) $\left(\mathrm{m}^{2} /\right.$ day), and

$v_{x}=$ average linear velocity $(\mathrm{m} /$ day $)$. 
The randomness of heterogeneous groundwater systems can be accounted for by adding a stochastic component to equation (7.4.1), and it can be given by

$$
\frac{\partial C}{\partial t}=\left\{D_{L}\left(\frac{\partial^{2} C}{\partial x^{2}}\right)+D_{T}\left(\frac{\partial^{2} C}{\partial y^{2}}\right)\right\}-v_{x}\left(\frac{\partial C}{\partial x}\right)+\xi(x, t),
$$

where $\xi(x, t)$ is described by a zero-mean stochastic process.

We multiple equation (7.4.2) by $d t$ throughout and, formally replace $\xi(x, t) d t$ by $\zeta(t)$. We can now obtain the stochastic partial differential equation as follows,

$$
d C=\left\{D_{L}\left(\frac{\partial^{2} C}{\partial x^{2}}\right)+D_{T}\left(\frac{\partial^{2} C}{\partial y^{2}}\right)\right\} d t-v_{x}\left(\frac{\partial C}{\partial x}\right) d t+\varsigma(t) .
$$

The two parameters to be estimated are $D_{L}$ and $D_{T}$ (while $v_{x}=0.5$ in this case). For the two parameter case, we can write the right hand side of equation (7.4.3) as follows:

$$
f\left(t, C, \theta_{1}, \theta_{2}\right)=a_{0}(C, t)+\theta_{1} a_{1}(C, t)+\theta_{2} a_{2}(C, t),
$$

where,

$$
\begin{gathered}
a_{0}(C, t)=-v_{x}\left(\frac{\partial C}{\partial x}\right)=-0.5\left(\frac{\partial C}{\partial x}\right) ; \quad a_{1}(C, t)=\frac{\partial^{2} C}{\partial x^{2}} ; \quad a_{2}(C, t)=\frac{\partial^{2} C}{\partial y^{2}} ; \\
\theta_{1}=D_{L} ; \quad \text { and } \quad \theta_{2}=D_{T} .
\end{gathered}
$$

The log-likelihood function can be written as (see Chapter 1),

$$
\begin{aligned}
l\left(\theta_{1}, \theta_{2}\right)= & \sum_{i=1}^{M} \int_{0}^{T}\left\{a_{0}\left(C_{i}, t\right)+\theta_{1} a_{1}\left(C_{i}, t\right)+\theta_{2} a_{2}\left(C_{i}, t\right)\right\} d C_{i}(t) \\
& -\frac{1}{2} \sum_{i=1}^{M} \int_{0}^{T}\left\{a_{0}\left(C_{i}, t\right)+\theta_{1} a_{1}\left(C_{i}, t\right)+\theta_{2} a_{2}\left(C_{i}, t\right)\right\}^{2} d t
\end{aligned}
$$

If we have values for $C(x, y, t)$ at $M$ discrete points in $(x, y)$ coordinate space for a period of time $t$ (where $0 \leq t \leq T$ ), then differentiating equation (7.4.5) with respect to $\theta_{1}$ and $\theta_{2}$, respectively, we get the following two simultaneous equations:

$$
\begin{aligned}
& \sum_{i=1}^{M} \int_{0}^{T} a_{1}\left(C_{i}, t\right) d C_{i}(t)-\sum_{i=1}^{M} \int_{0}^{T}\left\{a_{0}\left(C_{i}, t\right)+\theta_{1} a_{1}\left(C_{i}, t\right)+\theta_{2} a_{2}\left(C_{i}, t\right)\right\}\left\{a_{1}\left(C_{i}, t\right)\right\} d t=0 \\
& \sum_{i=1}^{M} \int_{0}^{T} a_{2}\left(C_{i}, t\right) d C_{i}(t)-\sum_{i=1}^{M} \int_{0}^{T}\left\{a_{0}\left(C_{i}, t\right)+\theta_{1} a_{1}\left(C_{i}, t\right)+\theta_{2} a_{2}\left(C_{i}, t\right)\right\}\left\{a_{2}\left(C_{i}, t\right)\right\} d t=0
\end{aligned} .
$$


We simplify equation (7.4.6) to

$$
\begin{aligned}
& \left\{\sum_{i=1}^{M} \int_{0}^{T} a_{1}\left(C_{i}, t\right) d C_{i}(t)-\sum_{i=1}^{M} \int_{0}^{T} a_{0}\left(C_{i}, t\right) a_{1}\left(C_{i}, t\right) d t\right\} \\
& -\theta_{1} \sum_{i=1}^{M} \int_{0}^{T}\left\{a_{1}\left(C_{i}, t\right)\right\}^{2} d t-\theta_{2} \sum_{i=1}^{M} \int_{0}^{T}\left\{a_{1}\left(C_{i}, t\right)\right\}\left\{a_{2}\left(C_{i}, t\right)\right\} d t=0 \\
& \left\{\sum_{i=1}^{M} \int_{0}^{T} a_{2}\left(C_{i}, t\right) d C_{i}(t)-\sum_{i=1}^{M} \int_{0}^{T} a_{0}\left(C_{i}, t\right) a_{2}\left(C_{i}, t\right) d t\right\} \\
& -\theta_{1} \sum_{i=1}^{M} \int_{0}^{T}\left\{a_{1}\left(C_{i}, t\right)\right\}\left\{a_{2}\left(C_{i}, t\right)\right\} d t-\theta_{2} \sum_{i=1}^{M} \int_{0}^{T}\left\{a_{2}\left(C_{i}, t\right)\right\}^{2} d t=0
\end{aligned}
$$

Now we substitute $a_{0}\left(C_{i}, t\right), a_{1}(C, t), a_{2}(C, t), \theta_{1}$ and $\theta_{2}$ in equations (7.4.7) to obtain the following set of equations:

$$
\begin{aligned}
& \left\{\sum_{i=1}^{M} \sum_{t=0}^{T}\left\{\frac{d^{2} C_{i}}{d x^{2}}\right\} d C_{i}(t)+0.5 \sum_{i=1}^{M} \sum_{t=0}^{T}\left\{\frac{d C_{i}}{d x}\right\}\left\{\frac{d^{2} C_{i}}{d x^{2}}\right\} d t\right\} \\
& -\theta_{1} \sum_{i=1}^{M} \sum_{t=0}^{T}\left\{\frac{d^{2} C_{i}}{d x^{2}}\right\}^{2} d t-\theta_{2} \sum_{i=1}^{M} \sum_{t=0}^{T}\left\{\frac{d^{2} C_{i}}{d x^{2}}\right\}\left\{\frac{d^{2} C_{i}}{d y^{2}}\right\} d t=0 \\
& \left\{\sum_{i=1}^{M} \sum_{t=0}^{T}\left\{\frac{d^{2} C_{i}}{d y^{2}}\right\} d C_{i}(t)+0.5 \sum_{i=1}^{M} \sum_{t=0}^{T}\left\{\frac{d C_{i}}{d x}\right\}\left\{\frac{d^{2} C_{i}}{d y^{2}}\right\} d t\right\} \\
& -\theta_{1} \sum_{i=1}^{M} \sum_{t=0}^{T}\left\{\frac{d^{2} C_{i}}{d x^{2}}\right\}\left\{\frac{d^{2} C_{i}}{d y^{2}}\right\} d t-\theta_{2} \sum_{i=1}^{M} \sum_{t=0}^{T}\left\{\frac{d^{2} C_{i}}{d y^{2}}\right\}^{2} d t=0
\end{aligned}
$$

We can rewrite equations (7.4.8) as,

$$
\begin{aligned}
& m_{1}-D_{L} k_{1}-D_{T} l_{1}=0 \\
& m_{2}-D_{L} k_{2}-D_{T} l_{2}=0
\end{aligned}
$$

Where $m_{1}=\sum_{i=1}^{M} \sum_{t=0}^{T}\left\{\frac{d^{2} C_{i}}{d x^{2}}\right\} d C_{i}(t)+0.5 \sum_{i=1}^{M} \sum_{t=0}^{T}\left\{\frac{d C_{i}}{d x}\right\}\left\{\frac{d^{2} C_{i}}{d x^{2}}\right\} d t$,

$$
m_{2}=\sum_{i=1}^{M} \sum_{t=0}^{T}\left\{\frac{d^{2} C_{i}}{d y^{2}}\right\} d C_{i}(t)+0.5 \sum_{i=1}^{M} \sum_{t=0}^{T}\left\{\frac{d C_{i}}{d x}\right\}\left\{\frac{d^{2} C_{i}}{d y^{2}}\right\} d t \text {, and }
$$




$$
l_{2}=\sum_{i=1}^{M} \sum_{t=0}^{T}\left\{\frac{d^{2} C_{i}}{d y^{2}}\right\}^{2} d t \quad .
$$

The two simultaneous equations in (7.4.9) can be solved to obtain the estimates of the unknown parameters, $D_{L}$ and $D_{T}$, for a two-dimensional groundwater system. The solutions of equations (7.4.9) are,

$$
\begin{aligned}
& D_{L}=\frac{m_{1} l_{2}-m_{2} l_{1}}{k_{1} l_{2}-l_{1}^{2}}, \\
& \text { and } \\
& D_{T}=\frac{m_{1} l_{1}-m_{2} k_{1}}{l_{1}^{2}-k_{1} l_{2}} .
\end{aligned}
$$

We have estimated the longitudinal and lateral dispersion coefficients for 100 realisations for each of $\sigma^{2}$ value chosen, and their mean values are given in Table 7.1.

The transverse dispersion coefficient is significantly less than the longitudinal dispersion coefficient for the flow length $[0,1]$ when $\sigma^{2}$ is very small but approaches approximately 0.5 of longitudinal dispersion coefficient when $\sigma^{2}$ increases (Figure 7.12). Comparing Table 7.1 with Table 4.9 , we see that the dispersion coefficient, therefore, the dispersivity, is smaller in 2 dimensions especially when $\sigma^{2}>0.01$. This needs to be expected as the lateral dispersion provides another mechanism of energy dissipation, thwarting the dispersion in the longitudinal direction.

\begin{tabular}{ccc}
\hline$\sigma^{2}$ & $D_{L}$ & $D_{T}$ \\
\hline 0.001 & 0.0251 & 0.0003 \\
0.005 & 0.0258 & 0.0012 \\
0.01 & 0.0264 & 0.0017 \\
0.02 & 0.0273 & 0.0027 \\
0.04 & 0.0293 & 0.0053 \\
0.05 & 0.0304 & 0.0072 \\
0.06 & 0.0314 & 0.0089 \\
0.08 & 0.0332 & 0.012 \\
0.1 & 0.0354 & 0.0145 \\
0.15 & 0.04 & 0.0197
\end{tabular}

Table 7.1. Estimated mean longitudinal and transverse dispersion coefficients using 100 concentration realisations from SSTM2d for each of $\sigma^{2}$ value. 


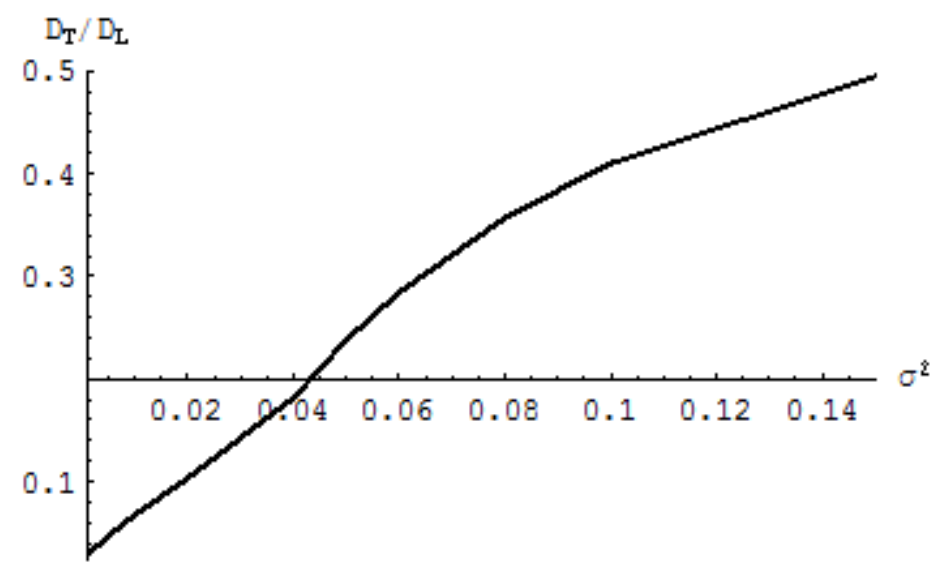

Figure 7.14. The ratio of the transverse dispersivity to the longitudinal dispersivity vs $\sigma^{2}$.

\subsection{Summary}

In this chapter, we developed the 2 dimensional version of SSTM for the flow length of $[0,1]$, and estimated the transverse dispersivity using the Stochastic Inverse Method (SIM) adopted for the purpose. The SSTM2d has mathematically similar form to SSTM but computationally more involved. However, the numerical routines developed are robust. We will extend SSTM2d in a dimensionless form to understand multi-scale behaviours of SSTM2d in the next chapter. 


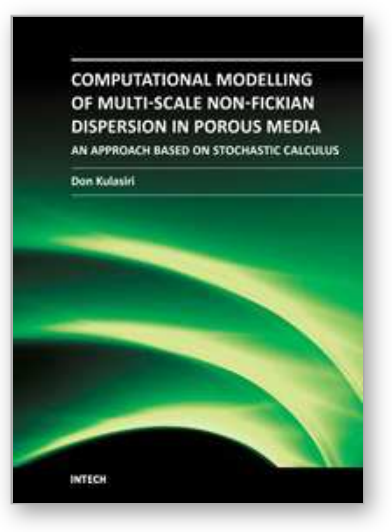

\author{
Computational Modelling of Multi-scale Solute Dispersion in \\ Porous Media - An Approach Based on Stochastic Calculus \\ Edited by
}

ISBN 978-953-307-726-0

Hard cover, 234 pages

Publisher InTech

Published online 04, November, 2011

Published in print edition November, 2011

This research monograph presents a mathematical approach based on stochastic calculus which tackles the "cutting edge" in porous media science and engineering - prediction of dispersivity from covariance of hydraulic conductivity (velocity). The problem is of extreme importance for tracer analysis, for enhanced recovery by injection of miscible gases, etc. This book explains a generalised mathematical model and effective numerical methods that may highly impact the stochastic porous media hydrodynamics. The book starts with a general overview of the problem of scale dependence of the dispersion coefficient in porous media. Then a review of pertinent topics of stochastic calculus that would be useful in the modeling in the subsequent chapters is succinctly presented. The development of a generalised stochastic solute transport model for any given velocity covariance without resorting to Fickian assumptions from laboratory scale to field scale is discussed in detail. The mathematical approaches presented here may be useful for many other problems related to chemical dispersion in porous media.

\title{
How to reference
}

In order to correctly reference this scholarly work, feel free to copy and paste the following:

Don Kulasiri (2011). The Stochastic Solute Transport Model in 2-Dimensions, Computational Modelling of Multi-scale Solute Dispersion in Porous Media - An Approach Based on Stochastic Calculus, (Ed.), ISBN: 978953-307-726-0, InTech, Available from: http://www.intechopen.com/books/computational-modelling-of-multiscale-solute-dispersion-in-porous-media-an-approach-based-on-stochastic-calculus/the-stochastic-solutetransport-model-in-2-dimensions

\section{INTECH}

open science | open minds

\author{
InTech Europe \\ University Campus STeP Ri \\ Slavka Krautzeka 83/A \\ 51000 Rijeka, Croatia \\ Phone: +385 (51) 770447 \\ Fax: +385 (51) 686166 \\ www.intechopen.com
}

\author{
InTech China \\ Unit 405, Office Block, Hotel Equatorial Shanghai \\ No.65, Yan An Road (West), Shanghai, 200040, China \\ 中国上海市延安西路65号上海国际贵都大饭店办公楼 405 单元 \\ Phone: +86-21-62489820 \\ Fax: $+86-21-62489821$
}


(C) 2011 The Author(s). Licensee IntechOpen. This is an open access article distributed under the terms of the Creative Commons Attribution 3.0 License, which permits unrestricted use, distribution, and reproduction in any medium, provided the original work is properly cited. 